Category: Neuromuscular junction disorders and channelopathies

\title{
RCT of Bumetanide in Hypokalaemic Periodic Paralysis (HypoPP) using abductor digiti minimi compound muscle action potential (CMAP) as an objective outcome measure
}

Renata S Scalco, Jasper Morrow, Andreea Manole, Iwona Skorupinska, Anna Bellin, Federico Ricciardi, Emma Matthews, Michael G Hanna, Doreen Fialho

MRC Centre for Neuromuscular Diseases, UCL, London, UK

\section{r.scalco@ucl.ac.uk}

Background: Treatment to abort acute attacks of weakness in patients with HypoPP is limited to potassium supplementation. Bumetanide inhibitor effect on the $\mathrm{Na}-\mathrm{K}-2 \mathrm{Cl}$ cotransporter may be a potential therapeutic agent based on mouse model studies.

Aims: To assess if $2 \mathrm{mg}$ bumetanide can abort an episode of focal hand weakness in patients with HypoPP.

Methods: RCT - ClinicalTrials.gov Identifier: NCT02582476

A focal attack of weakness was induced by hand rest following exercise (McManis protocol). Participants received either placebo or $2 \mathrm{mg}$ bumetanide on two different occasions at $40 \%$ decrement in abductor digiti minimi (ADM) compound muscle action potential (CMAP) amplitude from the maximum response. Electrophysiological measurements assessed the severity and the duration of the attack following $4 \mathrm{~h}$ of IMP intake.

Results: Nine participants completed both trial visits. There was no statistically significant difference in CMAP amplitude between the treatment groups at $1 \mathrm{~h}(p=0.27$, primary outcome). Two participants recovered from the attack of weakness ( $\leq 35 \%$ decrement in ADM CMAP amplitude from the maximum response) within 4 hours following Bumetanide intake; none recovered following placebo intake ( $\geq 40 \%$ decrement). There were no serious adverse events.

Conclusions: This is the first time bumetanide was utilised as a treatment option for patients with HypoPP. $2 \mathrm{mg}$ Bumetanide was safe but not effective to rescue a focal attack in an immobilised hand in the majority of patients. The McManis test, used here as a procedure and an outcome measurement in a clinical trial for the first time, was well tolerated. Data supports further studies of this agent. 